\title{
Design and Implementation of a Computer Aided Intelligent Examination System
}

\author{
https://doi.org/10.3991/ijet.v15i01.11102
}

\author{
Abdulrahman Abdullah Alghamdi, Mohammed Ateeq Alanezi, \\ Z. Faizal Khan ${ }^{(\bowtie)}$ \\ Shaqra University, Riyadh, Kingdom of Saudi Arabia \\ faizalkhanesu.edu.sa
}

\begin{abstract}
Developing a computer-based system for examinations is the substitute for the current examination system based on paper. In recent days, elearning has become more popular because of its adaptability, integrity and user friendliness. In terms of the paper-based examinations, the major challenge is the proctoring techniques used. In this paper, a novel method to avoid the presence of a proctor throughout the examination is proposed by an intelligencebased examination system. This method is proposed to improve e-learning using an intelligent question bank and an examination system. The system is designed with different complexity levels among the questions and it also acts as a tool for assessing the understanding of the student from the teaching materials. This system can be timesaving and more efficient with an adequate level of security. The proposed methodology can be classified into two main phases such as the design of question bank along with its database and the design of Artificial Intelligence (AI) based system for examination and its evaluation. Future works in this system can be done with the addition of theory-based questions and the integration of biometric based systems for enhancing the level of security.
\end{abstract}

Keywords - Information Systems, Artificial intelligence, E-Learning, Intelligent question bank system.

\section{Introduction}

Nowadays, E-Learning techniques have rapidly developed and there are many types of E-Learning based methodologies [1-4, 23] and Learning Management methods [7, 9-12] such as the traditional-based, online-based, and the workplace etc. [3-6, 24] as for education, through which everyone can perform the examinations. Information Technology (IT) has become an important role in the field of education. Elearning based on IT has become a necessary resource from which an instructor needs to handle the education because the instructor is the one who can develop the students by improving the skill of searching for resources, then to apply them to their day to day activities. E-Learning is a part of the learning activities [16, 17]. It is the easiest method of accessing the teaching materials, especially for the students. It is also a 
method for connecting the instructors to the students. By this e-learning based technology, the students can handle their homework using the internet. The instructor can conduct online-based examinations; and can evaluate and revert back the students with their marks.

At present, varieties of assessment and evaluation mechanisms are [6, 12, and 13] in the education system. In general, the majority of the instructors are conducting examinations based on the aptitude type of questions such as True-False type questions, Matching type of questions, Multiple Choice type questions or short answer type etc. For this process, each instructor needs to create the exam question paper manually, conduct the examination manually and then evaluate the answers manually. This manual process is tedious and it takes more time and effort to complete the examination and its evaluation process. Moreover, there may be a possibility of occurring errors during this evaluation. Hence the need of an automatized system for conducting and evaluating examinations is necessary.

The intelligent algorithm [15] will choose the already prepared test items, which are checked in advance for creating the question bank system in order to be the repository for examination. Some test items will be collected, and then will be chosen to be in the text based on the current test results. Furthermore, this system will use its already obtained score in order to access the ability of understanding of the student as well as to improve the examination. The question bank system can reduce the duration of test item production. As a result, it is an advantage for education: for example, there are some good test items, which enhance their standard and reliability. Artificial intelligence [4] will separate test items in many categories according to their difficulty. Moreover, it can choose test items to create exams according to learning objectives.

\section{Objectives of the Proposed Methodology} lows:

The main objects of the proposed intelligent based examination system are as fol-

- To have an insight into conducting and monitoring the examinations online among the students.

- To know various substructures which are used to examine the online based examination based on intelligent systems. This includes studying its operational characteristics and the working environment.

- To design an intelligent based methodology for conducting and evaluating it.

- Modification of the current examination system based on the proposed methodology of examination system.

The paper is organized as follows; section 2 depicts the literature review focusing on various methods proposed by earlier researchers, section 3 discusses the proposed methodology, its phased and the modules along with the system architecture, section 4 depicts the result and discussion section, conclusion and future works are shown in section 5 . 


\section{$3 \quad$ Literature Review}

Talent management (TM) has been considered as a process of human capital management which can be used to measure the activities of individual students [10]. It is a process of attracting the students who are able to do critical tasks in an organization ([2] Rana and Abbasi, 2013; [3] AlLozi et al., 2018). According to [4] Lewis and Heckman (2006), the TM can be categorized according to two classifications. Initially, it is the process of acquiring the students, training them and finding the individuals who are really talented. It can be a done to make sure of the number of talented students studying in the classroom. Lastly, classifying the students based on their level of performance and then providing them with prizes. The TM focuses on various factors such as, management of a student's performance, planning of their career and development of their leadership qualities.

Devi (2017) [6] did a work on the role of employees and their involvement in the $\mathrm{TM}$ process in the performance evaluation of an organizational. The TM consists of four factors [7]: the identification of talent by selecting employees for the positions of leadership in future, planning a strategy, which enables the talented persons to be prepared for positions in future, development of the talent through education and its consecutive trainings, and development of the career. It also focuses on managing the challenging tasks present in a job by motivation. Talents can also been measured based on Rees et al. (2013) [10] using the three factors: effectiveness, continuity and commitment. Various software and tools have been proposed by recent researchers for the efficient application of the employee evaluation in order to support the improvement. Examples for these various tools are management of knowledge, management of coordination, information and communication exchange between the employees. An online based task and employee management tool. Trello [8] provides various features in order to use it collaboratively. Various features such as notifications panel, calendar panel, comment window, file manager etc. were present in it. It can be used individually or in teams. All the users who are utilizing it can perform operations such as creating sets of checklists, addition of labels, inviting people to join in various tasks and operations, and also connecting with various other applications.

\subsection{Artificial intelligence based methods}

Artificial intelligence is the process of imitating human wisdom in the computer, in many tasks such as ability, logical reasoning, thinking, recognition etc. This methodology makes the computer perform as humans do [18], [19]. In this case, the computer can be the expert because it is trained and tested by the experts. This technology will solve this problem effectively.

\subsection{Machine learning based methods}

Machine learning is the process of training a computer to do the processes done by humans using an algorithm. This algorithm can be supervised or unsupervised. The 
machine learning algorithm is trained to predict the result, or to continue the process [20-22]. The computer can acknowledge the actual situation in order to decide effectively. Prediction of the result will be better according to the new data. Learning algorithms can be divided (according to learning processes) into four types: supervised learning, unsupervised learning, semi-supervised learning and reinforcement learning.

\subsection{Related works}

Authors in [7] developed a novel adaptive based assessment methodology to process examinations. Their methodology improved the quality of examination system [14] by creating an examination pattern based on the e-learning based system. In their system, they performed pre-assessments of the questions so the examination can be moderate as well as hard for the students. Depending on the pattern of the examination, answers are also prepared. Researchers in [9] proposed an assessment system which is adaptive in type with the combination of an examination database. In this approach, the efficiency of the examination can be increased by choosing the questions in multiple forms. This computer-assisted examination system reduced the work of creating examination as well as the proctoring. This method improves the quality of the evaluation process in education.

Multiple assessments with formulas are proposed by [11] in order to create serial examinations and also for evaluating continuously. Their method is based on multiple criteria. Their online based examination system can be used in the bank system. It can be gradually changed dynamically for other examination systems for the students' learning purposes.

\section{$4 \quad$ Proposed Methodology}

The proposed intelligent examination system consists of two phases and four modules:

- User Management

- Course Management

- Question and its answer Management

- Examination and its evaluation Management

The first phase is the user and Course management. In this phase, the users such as the instructor, students and the administrator were managed. This is explained in section 4.3 .

The second phase is the question and its answer Management, and the examination and its evaluation management. This system was designed using AI. Fuzzy logic based methods are applied logically to get the suitable intelligent question bank system and its evaluation framework. Overall architecture of the proposed methodology is shown in Figure 1 


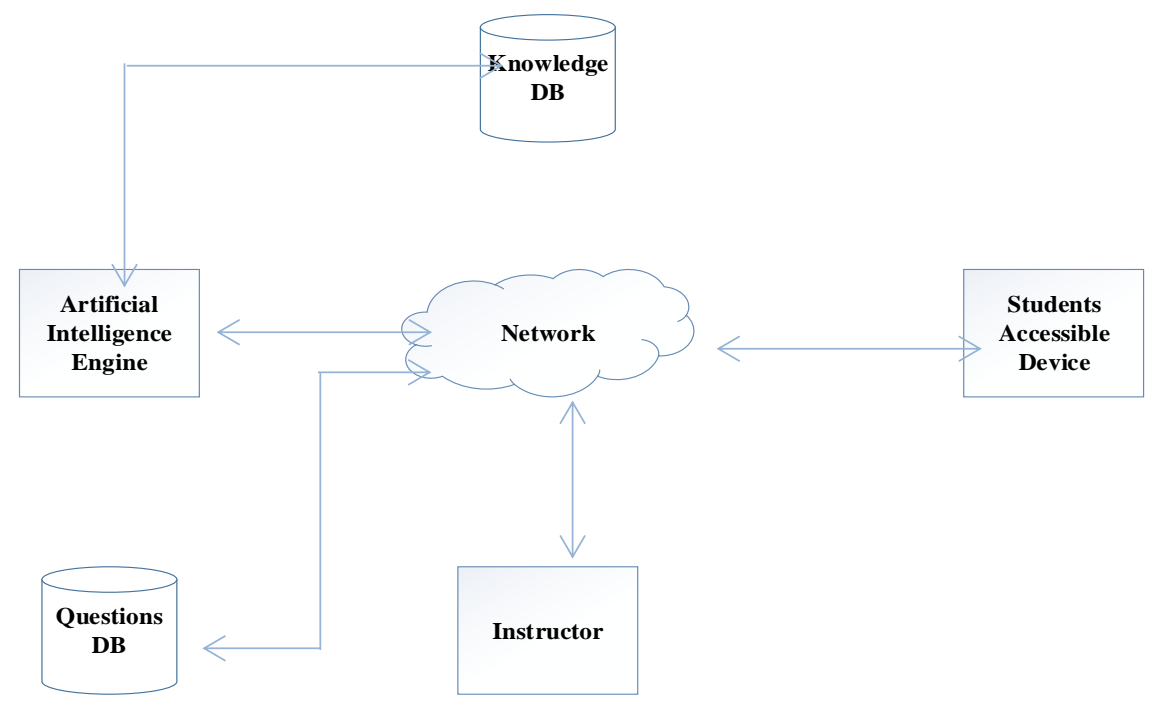

Fig. 1. Architecture of the Proposed Framework

\subsection{Working principle}

Working principle of the proposed system (shown in Figure 2) accepts the user request through an authentication system. The authentication system is directly connected with the users who are using this intelligent examination system. It accepts the user credentials such as the username and password from the respective users. Users such as the students, teacher and the administrator can access the system using the required credentials.

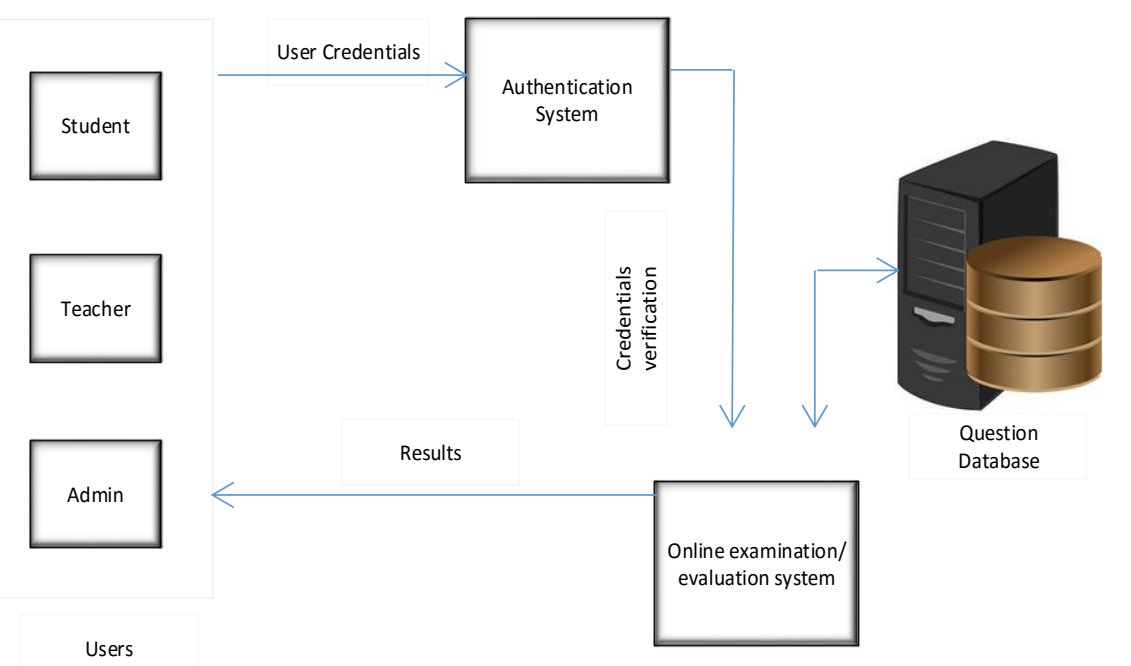

Fig. 2. Working principle of the proposed system 
The User interface agent is connected through an online based application. The protocols are defined for the users who are using the proposed system. All the policies are then parsed in the form of constraints and further stored in a centralized knowledge database. The database consists of users, roles and their permissions. The authentication system validates the username and password of the user. It sends the authenticated information to the examination /evaluation system. Roles were created by the authentication system for various operations including the permissions for particular functions. Roles are assigned to particular users and the assignments for those roles give the permissions to do the particular functions. Three different types of fields were present in the database, which are the student field, teacher information field and the admin Information field. The student field consists of all the information about the student such as the user Id, Password, University Id, subjects written, pass percentage, passed or failed subjects etc. The teacher information field consists of name, user Id, password, subjects, marks awarded etc. and finally, the admin field consists of all the username and passwords, access history etc.

\subsection{Intelligent question bank system}

The intelligent based question bank system consists of a set of 100 questions, which are created from to the students' syllabus, which can be a mixture of medium, easy and hard. Selection of the questions is automatized and intelligent based. The passing percentage of each level of exam is 70. Depending on the level of marks obtained in each level and in each question and the algorithm will choose the level of questions. The evaluation process is also automatized so that the algorithm can find the level of understanding of students in each section of the subject. The main advantage of the proposed method is analyzing the student's understanding level and mastery level in the respective chapters. The proposed methodology will adjust the questions based on the student's understanding level and mastery level.

The question database will have the questions uploaded by instructors. Each question has the following features:

- Aptitude type

- Question answers with relevancy to the correct answer for instance; correct answer will be $100 \%$ relevant, wrong answers would be $75 \%$ relevant, $50 \%$ relevant, $25 \%$ relevant and $0 \%$ relevant

- Question category / domain

- Question level: As medium, easy and hard

\subsection{Artificial intelligence engine}

Artificial intelligence based Classification process is used to determine the set of questions. This can be done using the Support Vector Machine algorithm. This is used in the question and answer management module in order to assign the suitable questions to the respective students. This is done by training and testing the proposed system. Selecting the questions can be done based on the difficulty levels medium, easy 
and hard. The questions are aptitude type. Question answers with relevancy to the correct answer will be $100 \%$ relevant and the wrong answers would be $75 \%$ relevant, $50 \%$ relevant, $25 \%$ relevant or $0 \%$ relevant depending on the correctness of the answer. Algorithm for assigning marks is as follows:

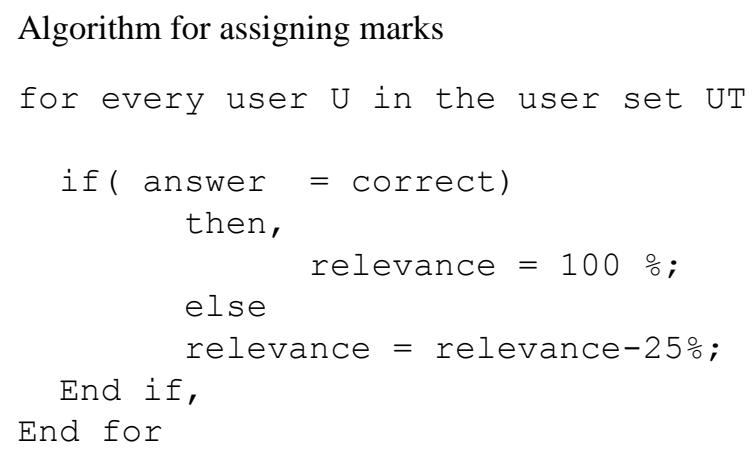

\subsection{User management}

The role of user management is to monitor functions such as login, editing the profile and setting permission for the users to access the system. Login function monitors the users who were accessing the system. It also maintains the correct username, password and sign up function before logging in to the system. Also, the Users can edit their profiles, by changing their password, email and their profile picture etc. It manages the setting permission to different types of users such as the student, teacher or administrator.

Table 1. Users and their role in the proposed system

\begin{tabular}{|c|l|l|}
\hline No. & \multicolumn{1}{|c|}{ Users } & \multicolumn{1}{|c|}{ Role } \\
\hline 1 & Instructors & $\begin{array}{l}\text { Maintain the overall system } \\
\text { Analyze the statistics of examination }\end{array}$ \\
\hline 2 & Students & $\begin{array}{l}\text { Perform the online examination } \\
\text { Verify the result }\end{array}$ \\
\hline 3 & AI Engine & $\begin{array}{l}\text { Make the complete process of examination and its evaluation as automatized } \\
\text { Maintain and manage the user accounts }\end{array}$ \\
\hline 4 & Administrators & Determine the permission of respective user \\
\hline
\end{tabular}

The proposed system consists of three types of users: instructors, students, and the administrators. Each user has different responsibilities. The types of users and their responsibilities are shown in Table 1 . The algorithm for accessing the system by a correct user and authenticating whether it is the correct user is as follows: 
Algorithm for Accessing the System

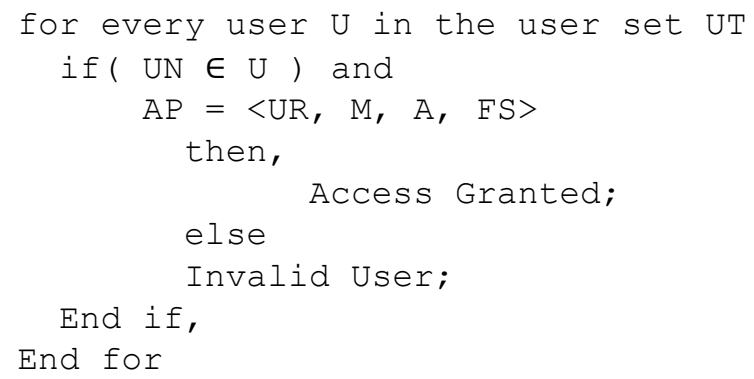

Algorithm for Authentication Policy

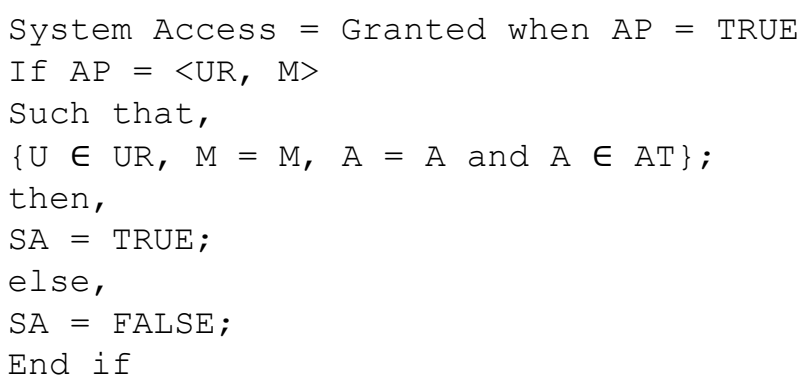

\subsection{Course management}

The role of user management is to monitor the course Management by adding and deleting the courses, setting the courses by Set items, such as grouping courses, evaluation rate calculation etc. and artificial intelligence for grouping the courses automatically based on the register numbers of the student.

\subsection{Question and answer management}

Question and its answer management monitors the questions and answers by adding, deleting and updating the questions and its corresponding answers, setting the questions, and assigning the answers. Artificial intelligence (AI) based methodology is used for grouping the questions and its answers automatically based on the register numbers of the student. Application of the proposed intelligent based question bank and examination system online can reduce the cost of budget, time taken, and trouble in managing the information technology, since the users do not need to buy hardware, software, or software updates, which are expensive and have long-time maintenance. When the number of users increases, it is easy to update the contents in the system. If we want to increase the storage space, it is easy to add extra storage in the server. 


\subsection{Examination and evaluation management}

Setting Examination, Monitoring Examination, Evaluation of Examination, Declaration of the results and suggestions, and an Artificial intelligence based methodology are the tasks done by the Examination and its evaluation Management system. Setting the examination comprises of setting the date, time for the starting and finishing the exam etc. Monitoring examination consists of checking the students who are performing or finished the exam. Declaration of the results and suggestions comprises of evaluating the examination and writing reports based on the results obtained. Applying AI based algorithm for choosing the overall examination and its evaluation management is also to be done.

Evaluation system: In this section, an Artificial Intelligence based intelligent system is used for allocating the marks for each question based on the correct answers. The algorithm is trained with questions and its respective answers. The AI agent is an intelligent module which allocates the correct answers for the appropriate questions and it also allocates the set of questions to a specific student based on the register number so that the question sets cannot be repeated among the others. The AI based subsystem can generate the questions, which are then sent to the device where the student can access the questions and answer them.

AI agent: An Artificial Intelligence based agent is an intelligent module which has the following tasks:

- Generate questions to be sent to students

- Generate a knowledge base from students' answers, with feedback to know more about some explanations of students' answers and areas of weakness and areas of strength

Student accessible device: Access the questions and answer them and submit the answers

Instructor: Instructor can do the following tasks:

- Add / Modify / Delete Question

- Generate exam with predefined number of questions and the percentage of each level of questions

The knowledge database Stores the generated knowledge from student answers feedback via AI engine. Algorithm for evaluating the students is as follows:

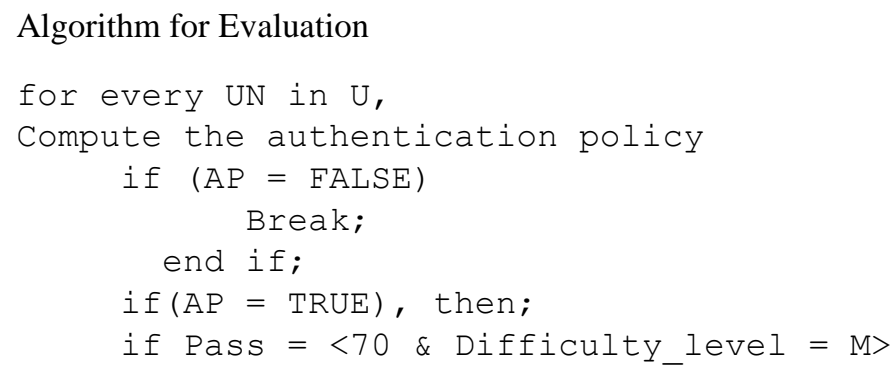




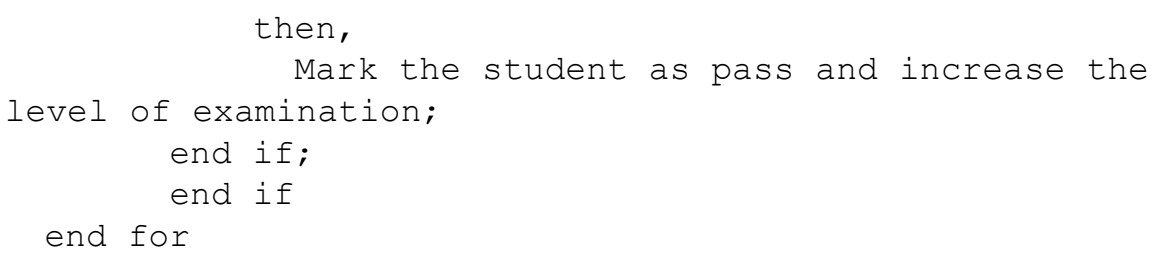

Terminology used: Following are the various terminologies used in the proposed Computer Aided Intelligent Examination System

- Action (A): Acton to be performed based on the user request.

- Action Type (AT): Set of all actions within the system

- User Set (UT): Set of users who can access the files in a file set present in the health database. (ex.) \{Admin, Student ....\}

- User Role (UR): Role of the user who is accessing the system.

- User Type (U): Type of the user who is accessing the system.

- User Credentials $(U C)$ : Username and password of the user who is going to access the system.

- Authentication Policy (AP): User Credentials for accessing the proposed examination system $\mathrm{AP}=\langle\mathrm{UR}, \mathrm{M}\rangle$

$\mathrm{M}$ is the mode of operation such as accessing, updating or deleting.

- System Access (SA): Accessing the proposed examination system by authenticated user.

$\mathrm{SA}=\langle\mathrm{U}, \mathrm{M}\rangle$

$\mathrm{U}$ is the user who made the request.

\section{$5 \quad$ Results and Discussion}

The proposed intelligent based examination system is implemented in such a way that it can be used online and verified for the accuracy. The figures shown below are the various interfaces from the executed system. 


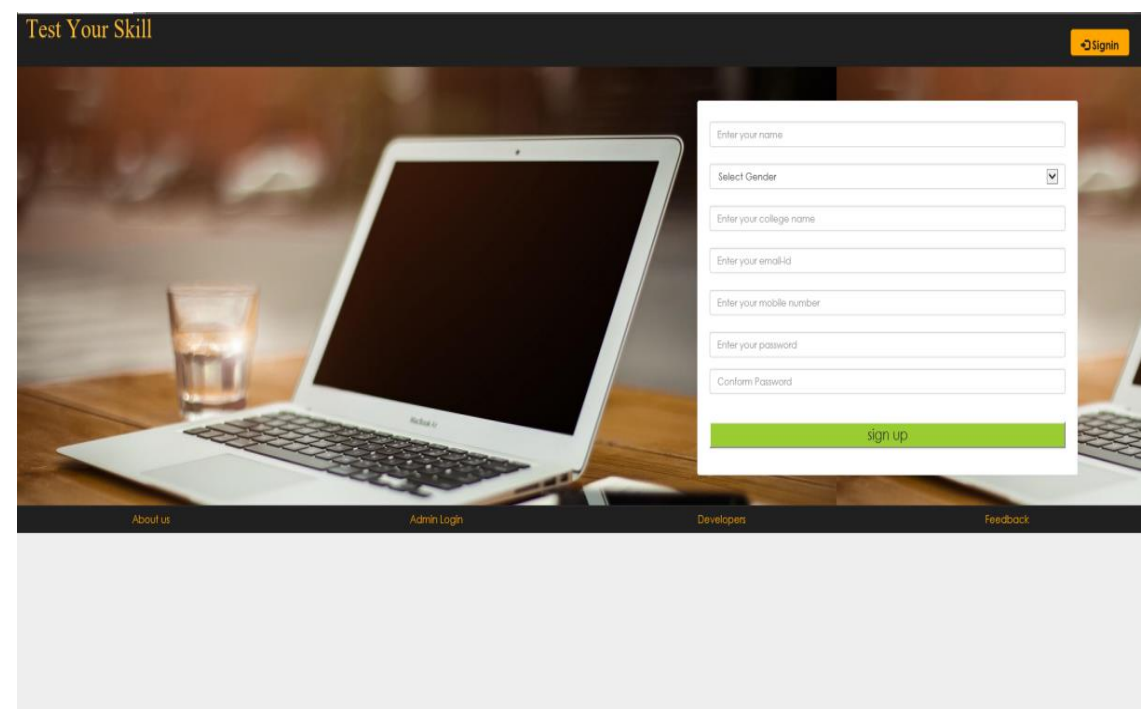

Fig. 3. Main Interface of the proposed intelligent examination system

Figure 3 shows the interface which loads whenever system is started. The user can enter the details as credentials in order to register in the system. It can be a student, or a teacher. This section leads to the authentication page.

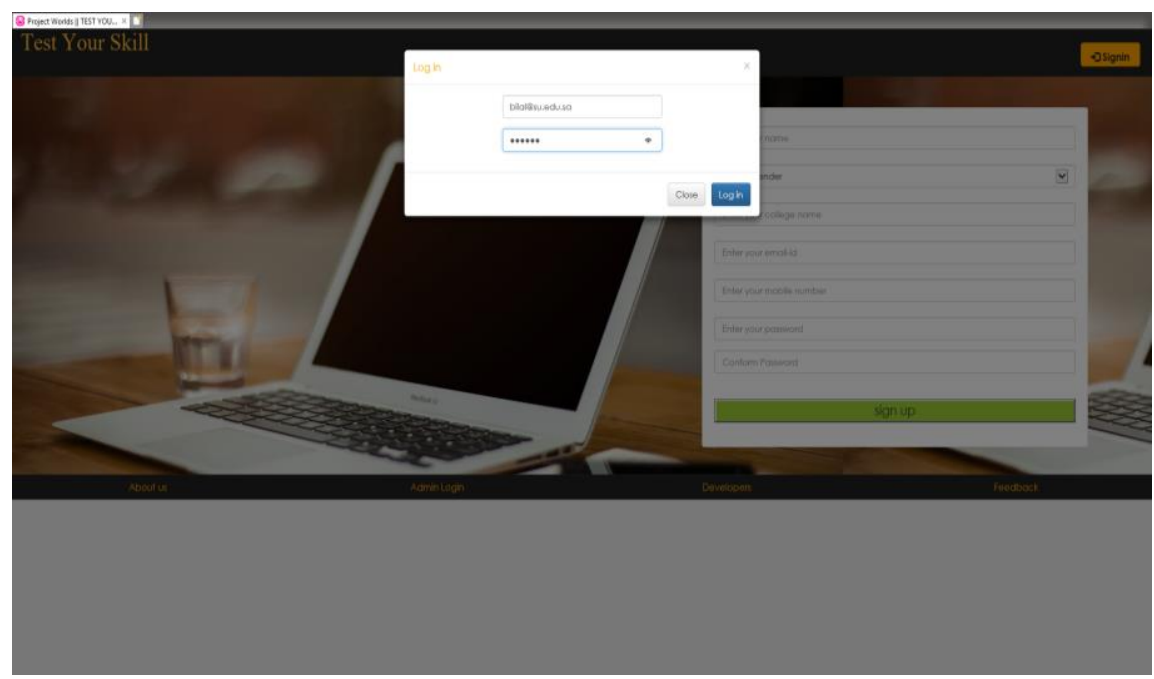

Fig. 4. User authentication interface

Figure 4 depicts the login page where the user can enter their user name and password credentials. If the user is a student, then they can enter their identity number (ID) and the password they used at the time of the registration. This page will give access to the user based on the type of user. If it is a student, then the student page 
will open and it will lead to the examination and marks section. If the user is a teacher, then the teacher page which contains the student's details, marks section etc. will be displayed.

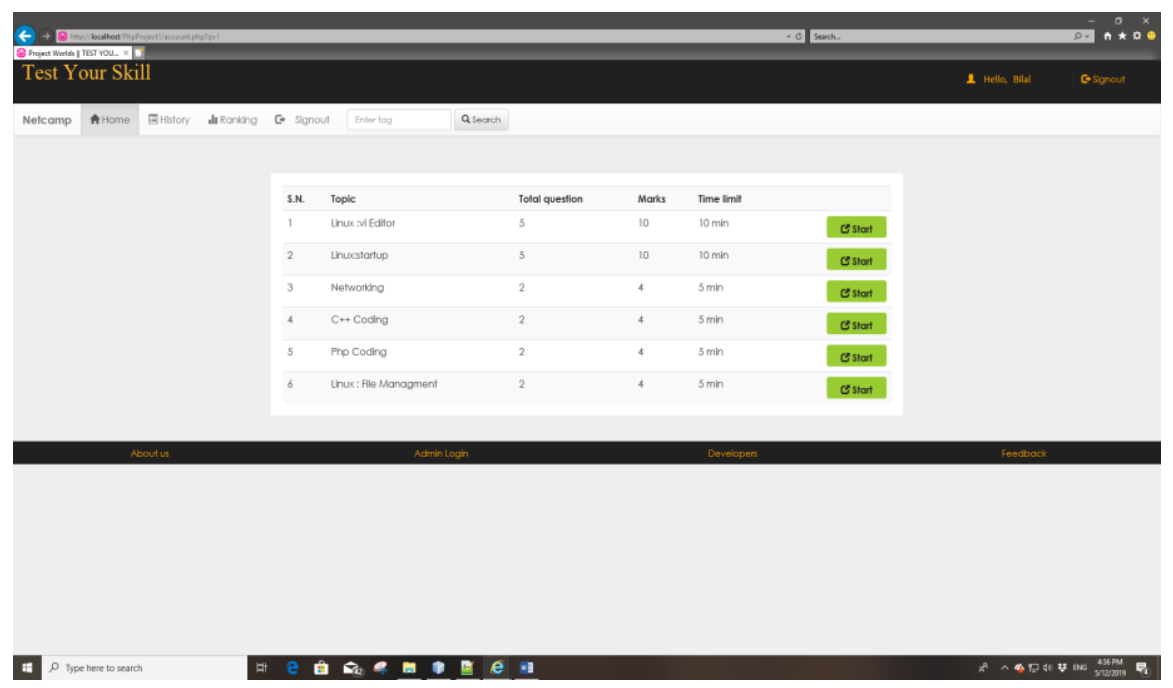

Fig. 5. Course selection interface

Figure 5 shows the interface for course selection under the students section. Each courser registered under the respective student ID number is shown in this page. The student can access his respective courses and start performing the examination by clicking the start button near the subjects.

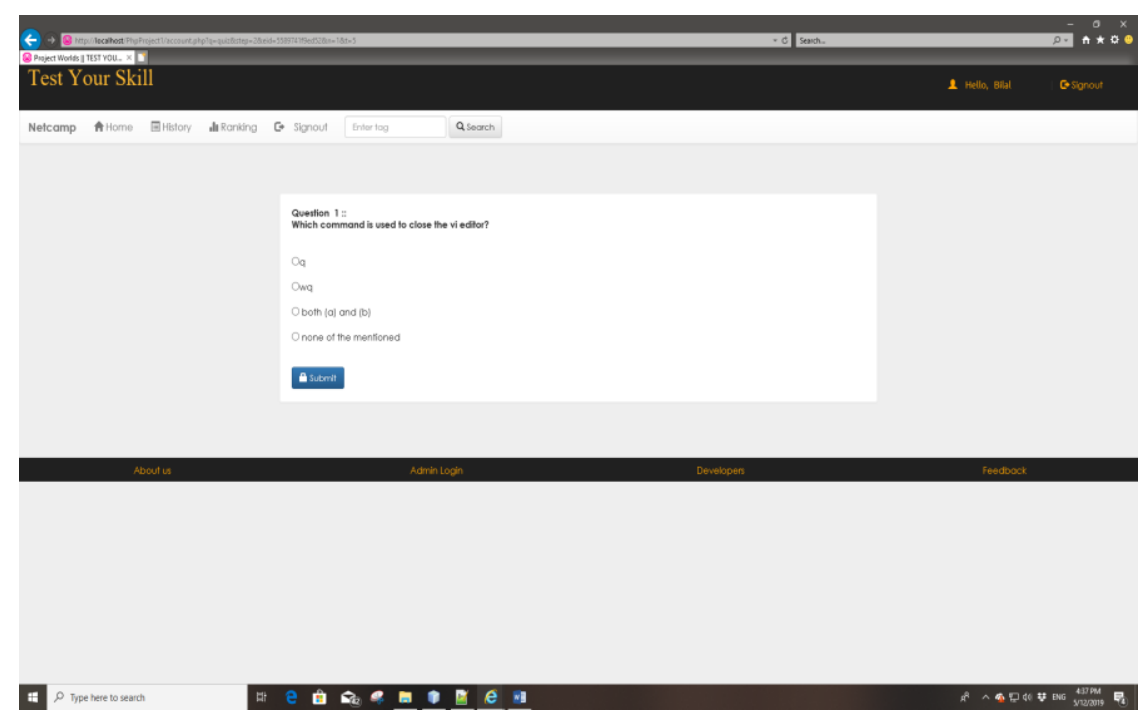

Fig. 6. Question interface 
Figure 6 displays the interface of a sample question paper from a subject which is registered under a student where the student can perform his examination. Setting of the questions is made randomly by the $\mathrm{AI}$ algorithm from the question bank present in the database. After answering the correct answer, the student can submit it using the submission button. Submitted answers are also stored in the database.

\section{Conclusion and Future Enhancements}

In this paper, a computer aided intelligent examination system is proposed for improving on e-learning based systems. The proposed system consists of four modules, which are the user management, course management, question and answer management and the examination and evaluation Management. An AI based algorithm is used for performing all the modules present in the proposed system. The proposed system is intelligent since it is trained and tested by the AI based system. Questions are formed based on the level of students and the marks are allotted based on the relevance of answers. The proposed system is more secure since it uses authentication procedures for the entire process. The entire system is designed in such a way that the examinations can be conducted more easily using the computer bases system. The AI based algorithm is applied to all of the four modules of the proposed system. Future works in this system can be done with the inclusion of theory-based questions and the integration of additional security measures such as the biometric based systems.

\section{$7 \quad$ Acknowledgement}

We would like to thank the Shaqra University for supporting this research. This work had been financed under Shaqra University, grant Nr. D180000/G01/N055.

\section{$8 \quad$ References}

[1] C. Whitehouse, "Item banks and on-demand tests," The Assessment and Qualifications Alliance (AQA), Centre for Education Research and Policy, pp. 1-4, 2012.

[2] Nasim Abdulwahab Matar, Defining E-Learning Level of Use in Jordanian Universities Using CBAM Framework, International Journal of Emerging. Technology in. Learning, Vol 12, No 3, pp. 142-153, 2017 https://doi.org/10.3991/ijet.v12i03.6497

[3] S. Banerjee, N. J. Rao, and C. Ramanathan, "Designing item banks in alignment with course outcomes for engineering courses," in Proc. IEEE 8th International Conference on Technology for Education, 2016. https://doi.org/10.1109/t4e.2016.039

[4] Quadri Noorulhasan Naveed, Naim Ahmad, Critical Success Factors (CSFs) for CloudBased e-Learning, International Journal of Emerging. Technology in. Learning, Vol 14, No 1, pp. 140-149, 2019 https://doi.org/10.3991/ijet.v14i01.9170

[5] K. Hammond, Practical Artificial Intelligence for Dummies, 1st ed. Narrative Science, 2015, pp. 1-30. 
[6] I.Portugal, P. Alencar, D. Cowan. (2015). The Use of Machine Learning Algorithms in Recommender Systems: A Systematic Review. [Online]. Available: https://arxiv.org/ftp/ arxiv/papers/1511/1511.05263.pdf

[7] K. Rajamani and V. Kathiravan, "An adaptive assessment system to compose serial test sheets using item response theory," in Proc. the 2013 International Conference on Pattern Recognition, Informatics and Mobile Engineering, 2013, pp. 120-124 https://doi.org/10. 1109/icprime.2013.6496458

[8] M. Wang, C. Wang, C. Lee, S. Lin, and P. Hung, "Type-2 Fuzzy set construction and application for adaptive student assessment system," in Proc. 2014 IEEE International Conference on Fuzzy Systems, 2014, pp. 888-894. https://doi.org/10.1109/fuzzieee.2014.689 $\underline{1894}$

[9] E. Khater, A. Hegazy, and M. E. Shehab, "Ontology-based adaptive examination system in e-learning management systems," in Proc. 2015 IEEE 7th International Conference on Intelligent Computing and Information Systems, 2015, pp. 243-250. https://doi.org/10.1109/ intelcis.2015.7397228

[10] Y. Cong, "Functional design of english online examination system based on ASP technology," in Proc. 3rd International Conference on Education, Management, Arts, Economics and Social Science, 2016, pp. 1140-1144. https://doi.org/10.2991/icemaess-15.2016.231

[11] N. Ketui, K. Homjun, K. Poonyasiri, J. Deepinjai, and P. Luekhong, "Item-based approach for online exam performance and its application," in Proc. 2016 13th International Conference on Electrical Engineering/Electronics, Computer, Telecommunications and Information Technology, 2016. https://doi.org/10.1109/ecticon.2016.7561297

[12] K. Mingsirithum and S. Sudsawad, "Development of online training kits to produce electronic books for teachers in the Secondary Educational Service Area Office 3," Bangkok: Sukhothai Thammathirat Open University, 2012.

[13] K. Kularbphettong, "An adaptive web-based intelligent tutoring using mastery learning and logistic regression techniques," Journal of 31-35, 2014.

[14] P. K. Gupta, "Mobile examination system," in Proc. 2012 2nd IEEE International Conference on Parallel, Distributed and Grid Computing, 2012, pp. 302-306.

[15] P. Lu, X. Cong, and D. Zhou, "The research on web-based testing environment using simulated annealing algorithm," The Scientific World Journal, pp. 1-12, 2014.

[16] M. Kang and W. S. Shin, "An Empirical Investigation of Student Acceptance of Synchronous e-Learning in an Online University," Journal of Educational Computing Research, vol. 52, no. 4, pp. 475-495, 2015 https://doi.org/10.1177/0735633115571921

[17] S. A. S. Salloum, "Investigating Students' Acceptance of e-Learning System in Higher Educational Environments in the UAE: Applying the Extended Technology Acceptance Model (TAM)," 2018

[18] R. A. S. Al-Maroof and M. Al-Emran, "Students Acceptance of Google Classroom: An Exploratory Study using PLS-SEM Approach," International Journal of Emerging Technologies in Learning (iJET), vol. 13, no. 6, pp. 112-123, 2018. https://doi.org/10.3991/ijet. v13i06.8275

[19] T. Almarabeh, "Students' Perceptions of E-learning at the University of Jordan," International Journal of Emerging Technologies in Learning (iJET), vol. 9, no. 3, pp. 31-35, 2014.

[20] S. S. Binyamin, M. J. Rutter and S. Smith, "The Influence of Computer Self-Efficacy and Subjective Norms on the Students' Use of Learning Management Systems at King Abdulaziz University," International Journal of Information and Education Technology, pp.693-699, 2018. https://doi.org/10.18178/ijiet.2018.8.10.1124 
[21] A. Janson, M. Söllner and J. M. Leimeister, "Individual Appropriation of Learning Management Systems - Antecedents and Consequences," AIS Transactions on HumanComputer Interaction, vol. 9, no. 3, pp. 173-201, 2017 https://doi.org/10.17705/1thci.00094

[22] K. Kabassi, I. Dragonas, A. Ntouzevits, T. Pomonis, G. Papastathopoulos and Y. Vozaitis, "Evaluating a Learning Management System for Blended Learning in Greek Higher Education," SpringerPlus, vol. 5, no. 101, pp. 1-12, 2016. https://doi.org/10.1186/s40064-016$\underline{1705-8}$

[23] Noorulhasan Naveed Quadri, AbulHafeez Muhammed, Sumaya Sanober, Mohamed Rafik N. Qureshi, Asadullah Shah, Barriers Effecting Successful Implementation of E-Learning in Saudi Arabian Universities, International Journal of Emerging. Technology in. Learning, Vol 12, No 6, pp. 94-107, 2017. https://doi.org/10.3991/ijet.v12i06.7003

[24] J. A. Akrimi, A. R. Ahmad, L. E. George, and S. Aziz, "Review of artificial intelligence," International Journal of Science and Research, vol. 2, no. 2, 2013.

\section{Authors}

Dr. Abdulrahman Alghamdi heads the computer sciences department of Shaqra University's College of Computing and Information Technology (CCIT). He is also a faculty member of the CCIT and the vice dean for development and quality. He has taught several university courses in the computer science field, including computer security, advanced programming, advanced database management systems, and artificial intelligence courses. His research interests include Information security, Programming, Database, and Image Processing.

Dr. Mohammed Alanezi, is an Associate Professor in the department of Computer Science in the College of Computing and Information Technology (CCIT) at Shaqra University where he has been since 2013. His research interests include EGovernment, E-Services, E-Health and Knowledge Management. He continued to contribute also to the development of various projects and systems; much of his work has been in embracing digital transformation and improving the current technology to solve challenges facing Shaqra University.

Dr. Z. Faizal khan is currently working as an Assistant professor at the College of Computing and Information Technology, Shaqra University in the Kingdom of Saudi Arabia. His research interests includes Artificial Intelligence, Intelligent based Systems, Image Processing and pattern Recognition. Currently he is working in a project financed under Shaqra University, grant Nr. D180000/G01/N055.

Article submitted 2019-06-24. Resubmitted 2019-08-08. Final acceptance 2019-08-12. Final version published as submitted by the authors. 